\title{
The distinct binding properties between avian/ human influenza A virus NS1 and Postsynaptic density protein-95 (PSD-95), and inhibition of nitric oxide production
}

Heng Zhang, Weizhong Li ${ }^{\dagger}$, Gefei Wang, Yun Su, Chi Zhang, Xiaoxuan Chen, Yanxuan Xu and Kangsheng Li

\begin{abstract}
Background: The NS1 protein of influenza A virus is able to bind with many proteins that affect cellular signal transduction and protein synthesis in infected cells. The NS1 protein consists of approximately 230 amino acids and the last 4 amino acids of the NS1 C-terminal form a PDZ binding motif. Postsynaptic Density Protein-95 (PSD-95), which is mainly expressed in neurons, has 3 PDZ domains. We hypothesise that NS1 binds to PSD-95, and this binding is able to affect neuronal function.

Result: We conducted a yeast two-hybrid analysis, GST-pull down assays and co-immunoprecipitations to detect the interaction between NS1 and PSD-95. The results showed that NS1 of avian influenza virus H5N1 (A/chicken/ Guangdong/1/2005) is able to bind to PSD-95, whereas NS1 of human influenza virus H1N1 (A/Shantou/169/2006) is unable to do so. The results also revealed that NS1 of H5N1 significantly reduces the production of nitric oxide (NO) in rat hippocampal neurons.
\end{abstract}

Conclusion: In summary, our study indicates that NS1 of influenza A virus can bind with neuronal PSD-95, and the avian $\mathrm{H} 5 \mathrm{~N} 1$ and human $\mathrm{H} 1 \mathrm{~N} 1$ influenza $\mathrm{A}$ viruses possess distinct binding properties.

Keywords: NS1, PSD-95, influenza virus, nitric oxide, neurons

\section{Background}

Influenza virus nonstructural protein (NS1) is encoded by a co-linear mRNA and consists of 202-237 amino acids, depending on the influenza A virus strains. The NS1 proteins contain an RNA-binding domain, an effector domain and an unstructured C-terminal domain around -20 amino acids long. The last 4 amino acids of the NS1 C-terminal compose the PDZ binding motif, which contributes to the virulence of influenza $A$ virus and modulates viral replication [1]. NS1 plays an important role in counteracting the cellular antiviral mechanism mediated by interferon (IFN) [2]. Both the protein kinase $R(P K R)$ and retinoic acid-inducible gene product I (RIG I) pathways are suppressed by NS1 $[3,4]$.

\footnotetext{
* Correspondence: ksli@stu.edu.cn

† Contributed equally

Department of Microbiology \& Immunology, Key Immunopathology Laboratory of Guangdong Province, Shantou University Medical College, Shantou 515041, PR China
}

(c) 2011 Zhang et al; licensee BioMed Central Ltd. This is an Open Access article distributed under the terms of the Creative Commons Attribution License (http://creativecommons.org/licenses/by/2.0), which permits unrestricted use, distribution, and reproduction in any medium, provided the original work is properly cited.
Postsynaptic density protein 95 (PSD-95) is a major scaffolding molecule localised at the postsynaptic density (PSD) of excitatory glutamatergic synapses and is mainly expressed in neurons of the hippocampus and cortex. PSD-95, which contains 3 PDZ domains, is a member of the membrane-associated guanylate kinase (MAGUK) family. PSD-95 binds with many postsynaptic membrane proteins, including $\mathrm{N}$-methyl-D-aspartate receptor (NMDAR), potassium channels, tyrosine kinases and cell adhesion molecules [7]. Both neuroligins and synaptic adhesion-like molecule (SALM) are able to interact with PSD-95 and balance neuronic excitation and inhibition $[8,9]$. The recruitment of the $\alpha$-Amino-3-hydroxy-5cells $[5,6]$. 
methyl-4-isoxazolepropionic Acid (AMPA) receptor at the synapse is affected by the expression level of PSD-95 [10]. The ionic equilibrium is mediated by PSD-95, and can regulate the expression of nitric oxide (NO) $[11,12]$. Furthermore, PSD-95 is also involved in many diseases, such as schizophrenia, autism and Fragile X Syndrome [13].

In our previous study, we found that microglia and astrocytes of the mouse cortex can be infected by avian and human influenza viruses in vitro, which result in the release of different levels of cytokines and NO [14]. It has also been demonstrated that acute encephalitis in mice is caused by infection with the neurovirulent influenza A virus, which can spread to the amygdale and hippocampus [15]. There was also difference in viral replication when mice brains were infected by neurovirulent $\mathrm{A} / \mathrm{WSN} / 33$ (H1N1) and nonneurovirulent $\mathrm{A} /$ Aichi/2/68 (H3N2) [16]. The physiological changes in neurons caused by direct infection with influenza virus or cytokines of microglia and astrocytes are unclear. Using the gene chip technique, it has been predicted that both avian and human viruses NS1 could bind to PDZ proteins [1]. Hongbing Liu has also reported that avian virus NS1 associates with the PDZ proteins Scribble, Dlg1, MAGI-1, MAGI-2 and MAGI-3, and reduces apoptosis during infection by disrupting Scribble's proapoptosis function [17]. However, it has not been shown that NS1 can bind to PSD-95, and the resulting neuronal changes are unclear. These results show that NS1 of the influenza A virus can bind to PSD-95. We also detected potential differences in binding properties between the avian influenza virus A/chicken/Guangdong/1/2005 (H5N1) and human influenza virus A/ Shantou/169/2006 (H1N1) NS1 proteins. We also measured the production of $\mathrm{NO}$ to investigate the influence of NS1/PSD-95 binding on signal transduction.

\section{Materials and methods}

Animals

This study was preapproved by the Ethical Committee of Shantou University Medical College and conducted in conformity with the Experimental Animal Management Bill issued on 14th November 1988 (Decree NO.2 of National Science and Technology Commission. China), and the National Institute of Health Guide for the Care and Use of Laboratory Animals (NIH Publication NO.80-23, revised 1996). One to two-day-old specific pathogen free (SPF) Sprague-Dawley rat were purchased from Shantou University Medical College Laboratory Animal Center, Shantou, Guangdong, China.

\section{Cells, viruses, and reagents}

Madin-Darby canine kidney (MDCK) cells were cultured in Dulbecco's modified Eagle's medium (DMEM) containing antibiotics and 10\% fetal calf serum. Primary neuron cells from rat were isolated and cultured in neurobasal medium supplemented with $10 \%$ fetal calf serum, $2 \% \mathrm{~B} 27$, and $1 \% \mathrm{~L}$-glutamine at $37^{\circ} \mathrm{C}$ in $5 \% \mathrm{CO}$.

Influenza A viruses $\mathrm{A} / \mathrm{Shantou} / 169 / 06(\mathrm{H} 1 \mathrm{~N} 1)$ and $\mathrm{A} /$ Chicken/Guangdong/1/05(H5N1) (abbreviated herein as ST169 and GD05, respectively) were used in this study.

Trizol reagent was purchased from Invitrogen (Carlsbad, CA, USA). AMV reverse transcriptase and Primer STAR HS DNA polymerase were purchased from TaKaRa (Dalian, China). Restriction endonucleases and T4 DNA ligase were purchased from New England Biolabs (NEB, Ipswich, MA, USA).

Mouse anti-NS1 antibody was purchased from Santa Cruz (Santa Cruz, CA, USA). Rabbit anti-PSD-95 antibody was purchased from Cell Signaling (Danvers, MA, USA). HRP-conjugated goat anti-mouse antibody was purchased from Sigma (St. Louis, MO, USA). HRP-conjugated goat anti-rabbit antibody, Cy3-labeled goat antirabbit antibody, and Alexa Fluor 488-labeled goat antimouse antibody were purchased from Beyotime Biotechnology (Jiangsu, China).

Yeast MATCHMAKER GAL4 two-hybrid system 3 and X- $\alpha$-gal were purchased from Clontech (Palo Alto, CA, USA); p-Nitrophenyl $\alpha$-D-galactopyranoside was purchased from Sigma (St. Louis, MO, USA). TNT T7 Quick Coupled Transcription/Translation Systems, Transcend Chemiluminescent Non-Radioactive Translation Detection Systems, and MagneGST pull down system were purchased from Promega (Madison, WI, USA). Protein G magnetic beads were bought from New England Biolabs (NEB, Ipswich, MA, USA). West Dura enhanced chemiluminescence reagents were purchased from Pierce (Rockford, IL, USA). A Total Nitric Oxide Assay kit was purchased from Beyotime Biotechnology (Jiangsu, China).

Plasmid pGST-PSD-95 harboring the full-length coding sequence of rat PSD-95 was kindly provided by $\mathrm{Dr}$ Bonnie L Firestein (Rutgers University in Piscataway). NS51-lentivirus was constructed and packaged by GeneChem (Shanghai, China).

\section{Primer design and plasmid construction}

Viral RNAs from different influenza A viruses were extracted using Trizol reagent, and the NS1 coding sequence was amplified by reverse transcription (RT)-PCR using the following primers: NS11-S1:5'-AATGGATCCATGGATTCCCACACTGT-3' and NS11-Al: 5'-TC GGGATCCTCAAACTTCTGACCTAAT-3' for ST169; NS51-S1: 5' -TATGGATCCATGGATTCCAACACTG TG-3' and NS51-Al: 5'-GACGGATCCTCAAACTTTTGACTCAATTG-3' for GD05. The underlined sequence indicates the $\mathrm{BamH} \mathrm{I}$ sites on the 5 'end of primers. PCR products were digested with $\mathrm{BamH} \mathrm{I}$ and inserted into 
pcDNA3 vector or PNF vector (a modified pcDNA3 vector with $\mathrm{N}$-terminal Flag tag) to yield recombinant plasmids pcDNA3-NS11, pcDNA3-NS51, PNF-NS11, and PNF-NS51. NS11 or NS51 represents the NS1 protein from $\mathrm{H} 1 \mathrm{~N} 1$ or $\mathrm{H} 5 \mathrm{~N} 1$ viruses.

To construct recombinant plasmids for yeast twohybrid assay, the following primers were designed: PSD-S1: 5'-CTGGAATTCATGGACTGTCTCTGTATAGT-3'; PSD-A1: 5'-AATGAATTCTCAGAGTCTC TCTCGGGC-3'; NS11-S2: 5'-ACTGAATTCATGGATTCCCACACTGTG-3'; NS51-S2: 5'-TATGGATCC TTATGGATTCCAACACTGTG-3'. EcoR I or BamH I sites on the 5'end of primers are underlined. PCR reaction was performed with plasmid pGST-PSD-95 as a template using primer sets PSD-S1 and PSD-A1. Reverse transcription PCR reactions were conducted using primer sets NS11-S2 and NS11-Al for ST169, and NS51-S2 and NS51-Al for GD05. PCR products were digested with appropriate enzymes and cloned into pGBKT7 or pGADT7 vector to generate plasmids pGBK-PSD95, pGAD-NS11, and pGAD-NS51, respectively.

All of the constructs were verified by sequencing.

\section{Yeast two-hybrid assay}

MATCHMAKER GAL4 two-hybrid system 3 was used for yeast two-hybrid assay according to the manufacturer's protocol. Briefly, to test the possible interactions between PSD-95 and NS1 proteins from different influenza viruses, AH109 yeast was transformed with plasmids pGAD-NS11, pGAD-NS51 or pGADT7 in combination with pGBK-PSD-95 and plated onto SD/Leu/-Trp media (DDO) and SD/-Ade/-His/-Leu/-Trp media (QDO). AH109 yeast transformed with plasmids pGBKT7-P53 plus pGADT7-T or pGBKT7-lam plus pGADT7-T served as a positive and negative control, respectively. The plates were incubated at $30^{\circ} \mathrm{C}$ for 3 days. Fresh AH109 colonies grown on DDO agar plates were picked and streaked on SD/-Ade/-His/-Leu/-Trp medium containing $\mathrm{X}-\alpha$-gal $(\mathrm{QDO} / \mathrm{X}-\alpha$-gal) followed by incubation at $30^{\circ} \mathrm{C}$. The growth and color of the colonies were observed daily for 2 days. In addition, single AH109 colonies grown on DDO agar plates were transferred into liquid DDO media and cultured at $30^{\circ} \mathrm{C}$ with shaking ( 250 cycles $/ \mathrm{min}$ ) for 36 hours. The supernatants were collected via centrifugation at 14,000 g for $2 \mathrm{~min}$ and used for $\alpha$-galactosidase activity analysis following the manufacturer's instructions.

\section{GST pull-down analysis}

Plasmids pGST-PSD95 or pGEX-5x-1 were transformed into Escherichia coli BL21. IPTG (isopropyl- $\beta$-D-thiogalactopyranoside) was added at a final concentration of $0.1 \mathrm{mM}$ at the mid-log phase. After $4 \mathrm{~h}$ of induction at $25^{\circ} \mathrm{C}$ with shaking $(200$ cycles $/ \mathrm{min})$, bacteria pellets were isolated by centrifugation at $12,000 \mathrm{~g}$ for $10 \mathrm{sec}$. Pellets were frozen and thawed 2 times, followed by lysis with MagneGST lysis reagent containing lyticase, DNase, and protease inhibitors for $40 \mathrm{~min}$. After centrifugation at $14,000 \mathrm{~g}$ for $10 \mathrm{~min}$, the precipitates were discarded and the supernatants were mixed with preequilibrated MagneGST beads at $4^{\circ} \mathrm{C}$ for $30 \mathrm{~min}$. Magnetic beads binding GST or GST-PSD-95 were washed with binding/wash buffer 5 times, and the bound proteins were separated by $8 \%$ SDS-PAGE gel and stained by Coomassie blue.

In vitro transcription/translation of NS11 or NS51 was performed using plasmids pcDNA3-NS11 or pcDNA3NS51 and TNT T7 Quick Coupled Transcription/Translation Systems, according to the procedure provided by manufacturer. The translated NS11 and NS51 proteins were detected by western blot using anti-NS1 antibody.

GST- or GST-PSD-95-binding beads were subsequently mixed with NS11 or NS51 proteins at room temperature for $2 \mathrm{~h}$. After 6 repetitions of extensive washing with binding/wash buffer containing $300 \mathrm{mM}$ $\mathrm{NaCl}$, the bound proteins were run on 12\% SDS-PAGE gel and blotted with antibody against NS1.

\section{Co-immunoprecipitation}

MDCK cells were transfected with plasmid PNF-PSD-95 or empty vector PNF for $36 \mathrm{~h}$. The cells were then infected with different influenza A viruses (ST169 or GD05) at an MOI of 2. Negative control cells were not infected. After $8 \mathrm{~h}$, cells were lysed in cold NP-40 lysis buffer on ice for $50 \mathrm{~min}$ and centrifuged at 14,000 $\mathrm{g}$ for $10 \mathrm{~min}$. Supernatants were collected and pre-cleared by protein G magnetic beads for $1 \mathrm{~h}$. Rabbit anti-PSD-95 antibody was added at a dilution of 1:100 for $2 \mathrm{~h}$. Normal rabbit IgG was used as a control. Protein-antibody mixtures were further incubated with protein $\mathrm{G}$ magnetic beads with gentle rotation overnight at $4{ }^{\circ} \mathrm{C}$. The beads were gathered and washed 3 times with NP-40 lysis buffer for $10 \mathrm{~min}$ each wash. The precipitated proteins were resolved on 10\% SDS-PAGE gel and probed with the rabbit anti-PSD-95 antibody (1:800) or mouse anti-NS1 antibody (1:1000) as well as HRP-conjugated goat anti-rabbit antibody (1:700) or HRP-conjugated goat anti-mouse antibody (1:2500). In addition, cell lysates were directly subjected to SDS-PAGE and western blotting using indicated antibodies.

\section{Western blotting analysis}

Protein extracts were fractionated by $10 \%$ SDS-PAGE gel and transferred onto nitrocellulose membranes. Proteins of interest were detected by incubating with antiPSD-95 (1:1000) or anti-NS1 (1:1000) in 5\% non-fat milk-TBST for $4 \mathrm{~h}$ at room temperature and blotting 
with HRP-conjugated secondary antibodies for $90 \mathrm{~min}$ followed by chemiluminescence detection.

\section{Nitric Oxide Detection}

Total Nitric Oxide Assay kit was used for NO detection according to the manufacturer's protocol. The NO was detected by measuring the nitrite using Griess reagent. To test the influence of the interaction between PSD-95 and NS1 proteins, the hippocampal neurons were infected by NS51-lentivirus for 3 days, then treated with acetylcholine (Ach) at $0.1 \mu \mathrm{mol} / \mathrm{mL}$. One day later, the cell lysates were centrifuged, and the NO level of the supernatant was detected.

\section{Results}

Detection of the interaction between NS1 and PSD-95 by yeast two-hybrid analysis

Firstly, to analyse the interaction of NS1 and PSD-95, the AH109 strains were transformed by using different plasmids. The results showed that all AH109 strains were able to grow in DDO. The strains transformed with pGAD-NS51 and pGBK-PSD-95 were able to grow on QDO, whereas the strains transformed with pGADNS11 and pGBK-PSD-95 were unable to grow on QDO (Figure 1-A). AH109 strains were picked from DDO and incubated on $\mathrm{QDO} / \mathrm{X}-\alpha$-gal. The results showed that the strains transformed with pGAD-NS51 and pGBKPSD-95 turned blue, and the color diffused around the colonies. Meanwhile, the strains transformed with pGAD-NS11 and pGBK-PSD-95 turned brown and showed the cessation of colonies (Figure 1-B). The data of $\alpha$-galactosidase activity revealed that AH109 strains transformed with pGAD-NS51 and pGBK-PSD-95 had a 3 -fold increase when compared with the positive control; the pGAD-NS11 and pGBK-PSD-95 strains looked similar to the negative control (Figure 1-C).

\section{Interaction of NS51 with PSD-95 in vitro}

Next, to detect the interaction of NS1 with PSD-95 in vitro, GST-PSD-95 or GST was immobilised on magnetic protein $\mathrm{G}$ beads, and then detected by Coomassie blue staining (Figure 2-A). Meanwhile, NS11 and NS51 proteins were transcribed and translated in vitro (Figure 2-B). NS11 or NS51 was incubated with GST or GSTPSD-95 immobilised onto magnetic protein $G$ beads (Figure 2-C). The results indicated that NS51 was able to precipitate with PSD-95, whereas NS11 was unable to bind PSD-95 in vitro.

\section{Interaction of NS51 with PSD-95 in vivo}

To further study the interaction between NS1 and PSD95 in vivo, co-immunoprecipitation and western blot assays were performed. If PSD-95 bound to NS1, this could be established by the resolving the $95 \mathrm{kDa}$ PSD95 proteins and the $26 \mathrm{kDa}$ NS1 proteins on an SDSPAGE gel. NS51 and PSD-95 were detected by western blotting (Figure 3-A), whereas no band for NS11 was detected (Figure 3-B).

\section{Production of NO while NS1 is bound to PSD-95}

To analyse the influence of this binding, NO was measured by enzyme standard instrument. The data revealed that the production of NO was reduced when hippocampal neurons were infected by NS51-lentivirus. Data represent the mean $\pm \mathrm{SD}$. The result suggests that the binding of NS51 and PSD-95 is able to inhibit the release of NO induced by Ach (Figure 4).

\section{Discussion}

The NS1 protein of influenza A viruses acts as a virulence factor that has multiple accessory functions during viral infection. The major role of NS1 is its inhibition of the host immune response, including the IFN, PKR and RIG 1 pathways. However, it has also been known that NS1 modulates the viral RNA replication, virus protein synthesis, and host cell physiology [18]. The PSD-95 protein contains $3 \mathrm{PDZ}$ domains, and the PDZ domains are bound to many postsynaptic membrane proteins, including NMDAR, potassium channels, tyrosine kinases and cell adhesion molecules. Around the NMDAR, many important proteins exist, such as neuronal nitric oxide synthase (nNOS), SynGAP and SPAR. As a scaffold protein, PSD-95 plays a very important role in neurons.

We found that there is an interaction between NS1 of influenza A virus and PSD-95 of hippocampal neuron, which has not been previously reported. PDZ domains are ubiquitous protein interaction modules that recognise a specific $\mathrm{C}$-terminal sequence to assemble protein complexes in multicellular organisms [19]. The binding of NS1 to PSD-95 may be through the PDZ domains of PSD-95 and the PDZ binding motif of the NS51 Cterminal. Our results also showed that only NS51 is able to bind with PSD-95. These results were concordant with the recent reports that avian virus NS1 and human virus NS1 have distinct binding properties [1]. It has been demonstrated that the NS1 proteins of H1N1, H3N2, H5N1, and H7N7 have different RNA silencing suppression activities, and that the short hairpin (shRNA) is mainly suppressed by the NS1 of A/WSN/33 (H1N1) [20]. It has also been indicated that NS1 has interactions with cleavage and polyadenylation specificity factor (CPSF); the NS1 of A/Tx/36/91 (H1N1) and udorn72 (H3N2) are able to bind with CPSF, whereas the NS1 of A/PR/8 fails to do so [21,22]. Obenauer et al. indicated that the distinct binding properties between avian and human NS1 are due to the differences in the NS1 C-terminal sequence. The NS1 PDZ binding motif of avian virus consists of ESEV, and the last 4 amino acids of human virus NS1 are the RSKV [1]. It was also reported that the full-length avian virus NS1 protein 


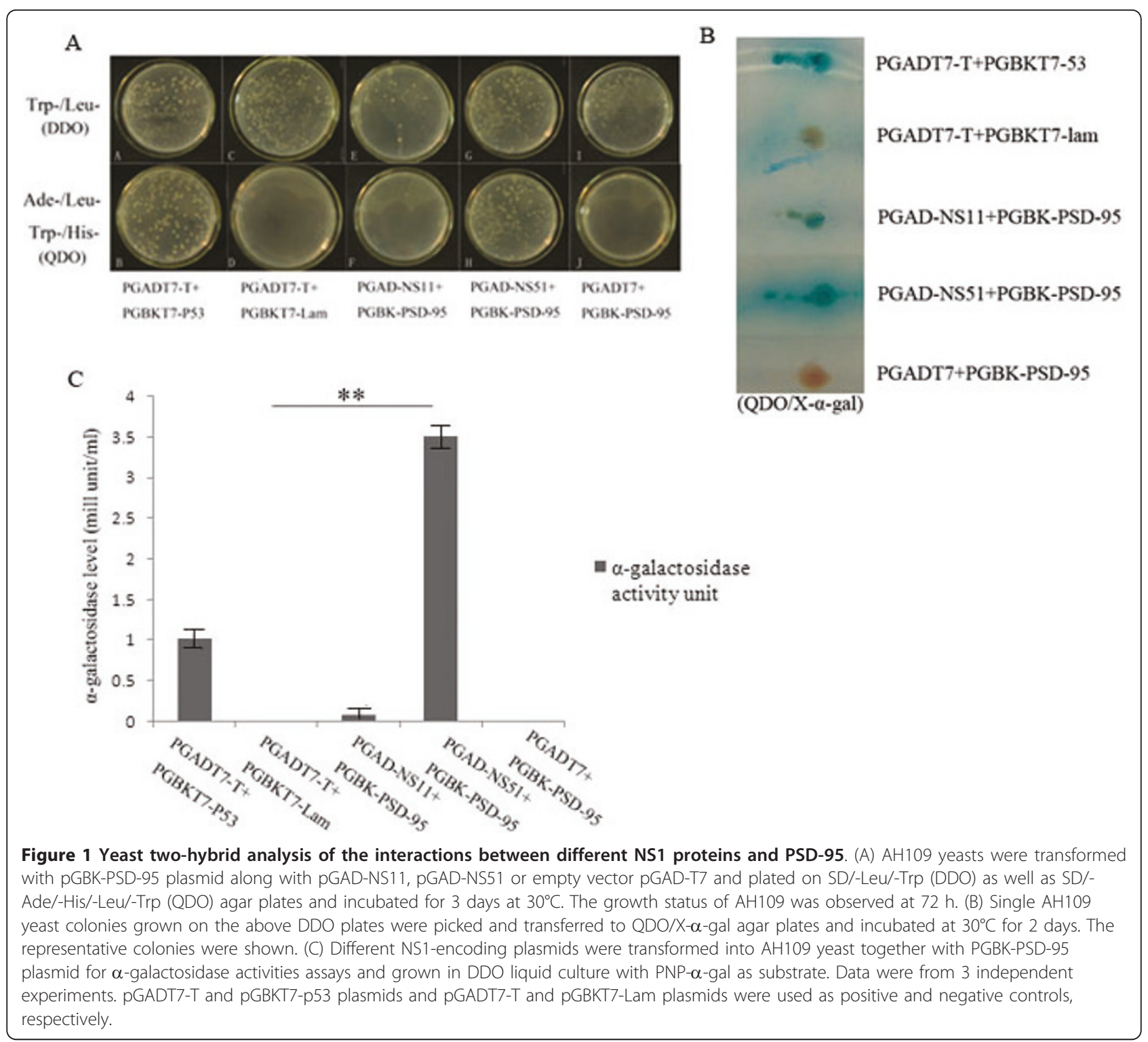

could bind to 30 different human PDZ domain containing proteins using the gene chip technique, whereas the human virus NS1 protein did not bind as many [1].

In our previous study, we reported that higher levels of cytokines and inflammatory mediators are released when astrocytes and microglia are infected by avian flu virus, but not when they are infected by human flu virus [14]. The virus infected neurons, and neighbouring uninfected neurons display apoptotic neurodegeneration, as shown by the immunohistochemistry [23]. It has been confirmed that the last 4 amino acids of the NS1 $\mathrm{C}$-terminal are the PDZ binding motif, which modulates the virulence in different strains of viruses [1,24]. When the 4 amino acids in the $\mathrm{C}$-terminal are deleted from NS1, the virus A/WSN/33 (H1N1) is severely attenuated in tissue culture, and increases in mean lethal dose (MLD50) compared with wild-type A/WSN/33 [25]. In addition, the functions of PSD-95 are involved in the clustering of several neurotransmitter receptors, adhesion molecule ionic channels, cytoskeletal elements, and signalling molecules at postsynaptic sites [13]. It has been demonstrated that PSD-95-mediated $\mathrm{K}^{+}$channel clustering is inhibited while PSD-95 function is reduced. The changes in $\mathrm{Ca}^{2+}$ are modulated by the NMDAR, the expression of which is affected by PSD-95, and the $\mathrm{Ca}^{2+}$ influx via glutamate-gated calcium channels regulates the formation of nitric oxide $[11,12]$. Therefore, NS51 might affect the neuron's functions through its interaction with PSD-95 and increase the infectivity of influenza A virus. 


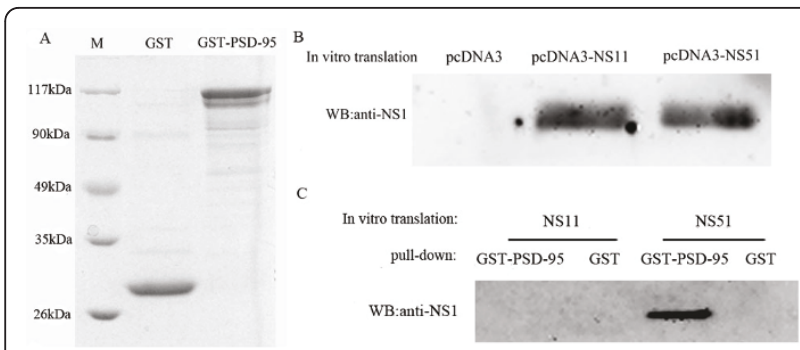

Figure 2 Differential binding of different NS1 proteins with PSD-95 in vitro. (A) GST or GST-PSD-95 proteins were expressed in E. coli BL21 and immobilised on magnetic beads. The immobilised GST or GST-PSD-95 proteins were separated in 8\% SDS-PAGE gel and detected by Coomassie blue staining. M stands for molecular weight standard. (B) NS11 or NS51 were translated in vitro using plasmids pcDNA3-NS11 or pcDNA3-NS51 and the TNT T7 Quick Coupled Transcription/Translation Systems. Expression of NS11 and NS51 was comfirmed by western blotting using anti-NS1 antibody. (C) NS11 or NS51 was incubated with GST or GST-PSD-95 immobilised onto magnetic beads. After extensive washing, bound proteins were dissociated from beads and subjected to western blotting analysis with anti-NS1 antibody.

To further evaluate the influence of the interactions, we measured the changes of $\mathrm{NO}$ in rat hippocampal neurons, which showed that $\mathrm{NO}$ was inhibited when rat hippocampal neurons were infected by NS51-lentivirus. Overexpression of PSD-95 in hippocampal neurons causes the dendritic spines to grow 2-3 times larger than normal size and increases the amount of nitric oxide synthase at postsynaptic densities [26]. NO is an important bioregulatory molecule produced by $\mathrm{nNOS}$ in

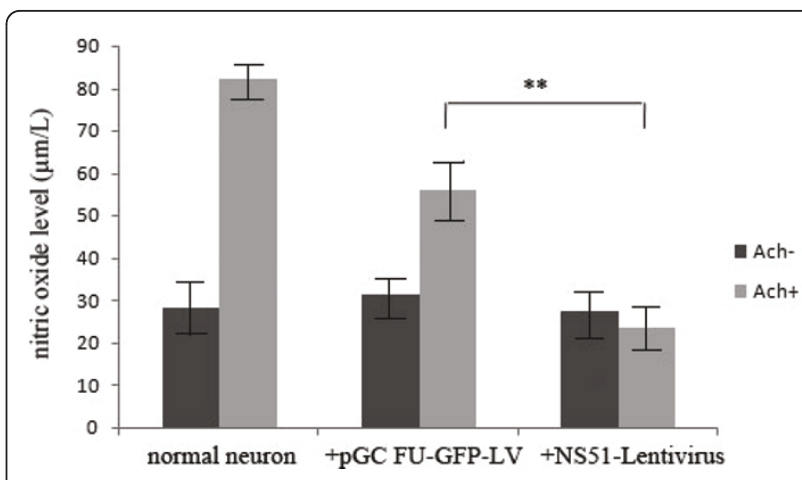

Figure 4 Production of NO while NS1 is bound to PSD-95 in hippocampal neurons. Hippocampal neurons were cultured for 3 days then infected by NS51-lentivirus or the control virus PGC FUGFP-LV. At 3 days post-infection, the cells were treated with Ach for $24 \mathrm{~h}$ and the release of $\mathrm{NO}$ was measured. Data were from 3 independent experiments. Relevant levels of significance are illustrated in the figure as ${ }^{*}$ for $p<0.01$. NS51-lentivirus and pGC FU-GFP-LV were used as positive and negative controls, respectively.

neurons. It has been reported that NO contributes to the activation of protein kinase $\mathrm{G}$ and the suppression of caspase activity $[27,28]$. NO can also induce apoptosis in the early stage of cells [29]. The binding of NS51 to PSD-95 leads to the reduction of NO, which might inhibit apoptosis and contribute to the replication of influenza A virus. In addition, as a free radical, NO is associated with cell aging and death, and is involved in the oxidative stress reaction in neurons. The reduction of NO induced by NS51 shows protective effects on

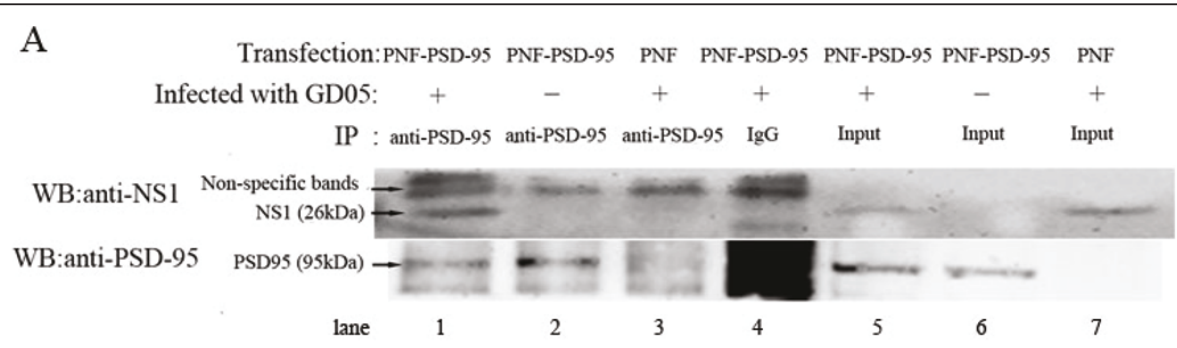

B

Transfection: PNF-PSD-95 PNF-PSD-95 PNF PNF-PSD-95 PNF-PSD-95 PNF-PSD-95 PNF Infected with ST169: $\quad+\quad+\quad+\quad+\quad+\quad+\quad+\quad+$

IP: anti-PSD-95 anti-PSD-95 anti-PSD-95 IgG Input Input Input

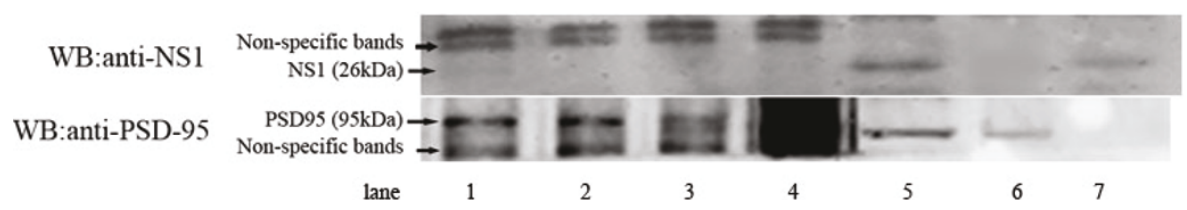

Figure 3 Differential interactions of different NS1 proteins with PSD-95 in mammalian cells. MDCK cells were transfected with either PNFPSD-95 plasmid or empty vector PNF. At $36 \mathrm{~h}$ post-transfection, the cells were infected or not infected with influenza virus GD05 (H5N1) (A) or ST169 (H1N1) (B) at an MOI of 2 for 8 h. Soluble cellular lysates were immunoprecipitated with rabbit anti-PSD-95 antibody (1:100) or normal rabbit lgG. The precipitated proteins or total cell lysates (input) were resolved by SDS-PAGE and blotted with antibodies against NS1 or PSD-95. Non-specific bands are indicated. 
central nervous system diseases induced by free radicals. The changes to NO are involved in many diseases, such as stroke, epilepsy, Alzheimer's disease, Huntington's disease, and Parkinson's disease [30,31].

\section{Conclusion}

In conclusion, we detected differences between avian and human influenza A virus NS1 binding to PSD-95. The NS1 of avian virus GD05 is able to bind with PSD95, but the NS1 of human virus ST169 is not. Additionally, the release of NO is reduced while NS51 is bound to PSD-95 in neurons. The interaction of NS1 with PSD-95 may help explain the mechanism that modulates the effect of NS1 on the host cell. The reduction of NO also implies that changes in neuron physiology are mediated by the binding of NS1 to PSD-95.

\section{Acknowledgements \\ This study was supported by National Natural Science Foundation of China (81001322, 30972766), Guangdong Natural Science Foundation \\ (9451503102003499), Specialized Research Fund for the Doctoral Program of Higher Education (20094402110004), State Key Lab of Agriculture Microbiology Open Foundation (AML200910), Shantou University Medical College Research Foundation, 211 Project of Guangdong Province (Mechanism and Prevention of Emerging infectious Diseases). We thank Dr Bonnie L Firestein for kindly providing the plasmid PSD-95.}

\section{Authors' contributions}

KSL and WZL contributed to project design and supervised the project. $\mathrm{H}$. Zhang carried out the majority of experiments in the study and drafted the manuscript. GFW, YS, CZ, XXC and YXX helped to carry out the experiments and draft the manuscript. All authors read and approved the final manuscript.

\section{Competing interests}

The authors declare that they have no competing interests.

Received: 1 April 2011 Accepted: 13 June 2011 Published: 13 June 2011

\section{References}

1. Obenauer JC, Denson J, Mehta PK, Su X, Mukatira S, Finkelstein DB, Xu XQ, Wang JH, Ma J, Fan YP, Karen MR, Robert GW, Erich H: Large-scale sequence analysis of avian influenza isolates. Science 2006, 311:1576-1580.

2. Wang X, Li M, Zheng H, Muster T, Palese P, Beg AA, Adolfo GS: Influenza A virus NS1 protein prevents activation of NF-kappaB and induction of alpha/beta interferon. J Virol 2000, 74:11566-11573.

3. Min JY, Li S, Sen GC, Krug RM: A site on the influenza A virus NS1 protein mediates both inhibition of PKR activation and temporal regulation of viral RNA synthesis. Virology 2007, 363:236-243.

4. Guo Z, Chen LM, Zeng H, Gomez JA, Plowden J, Fujita T, Katz M, Donis RO: NS1 protein of influenza A virus inhibits the function of intracytoplasmic pathogen sensor, RIG-I. Am J Respir Cell Mol Biol 2007, 36:263-269.

5. Ehrhardt C, Wolff T, Pleschka S, Planz O, Beermann W, Bode JG: Influenza A virus NS1 protein activates the PI3K/Akt pathway to mediate antiapoptotic signaling responses. J Virol 2007, 81:3058-3067.

6. Schultz C, Naomi S, Neumann G, Yoshihiro K, Hinshaw S: Influenza Virus NS1 Protein Induces Apoptosis in Cultured Cells. J Virol 2001, 75:7875-7881.

7. Morgan S: The postsynaptic NMDA-receptor-PSD-95 signaling complex in excitatory synapses of the brain. J Cell Sci 2001, 114:7875-7881.

8. Irie M, Hata Y, Takeuchi M, Ichtchenko K, Toyoda A, Hirao K, Takai Y, Rosahl TW, Sudhof TC: Binding of neuroligins to PSD-95. Science 1997, 277:1511-1515.

9. Wang CY, Chang K, Petralia RS, Wang YX, Seabold GK, Wenthold RJ: A novel family of adhesion-like molecules that interacts with the NMDAreceptor. J Neurosci 2006, 26:2174-2183.
10. Schnell E, Sizemore M, Karimzadegan S, Chen L, Bredt DS, Nicoll RA: Direct interactions between PSD-95 and stargazin control synaptic AMPA receptor number. Proc Natl Acad Sci 2002, 99:13902-13907.

11. Lin Y, Skeberdis VA, Francesconi A, Bennett MV, Zukin RS: Postsynaptic density protein-95 regulates NMDA channel gating and surface expression. J Neurosci 2004, 24:10138-10148.

12. Lin Y, Jover-Mengual T, Wong J, Bennett MV, Zukin RS: PSD-95 and PKC converge in regulating NMDA receptor trafficking and gating. Proc Natl Acad Sci 2006, 103:19902-19907.

13. Dove K, Alaa EH: Excitation control: balancing PSD-95 function at the synapse. Mol Neurosci 2008, 1:4

14. Wang GF, Zhang J, Li WZ, Xin G, Su Y, Gao Y, Zhang H, Lin G, Jiao X, Li KS: Apoptosis and proinflammatory cytokine responses of primary mouse microglia and astrocytes induced by human H1N1 and avian H5N1 influenza viruses. Cell Mol Immun 2008, 5:113-120.

15. Isamu M, Fumi G, Tetsuro K, Naoki K, Tsuyoshi S, Tomoaki Y, Takashi Y, Yukihiro N, Yoshinobu K: Differential activation of the c-Jun N-terminal kinase/stress-activated protein kinase and p38 mitogen-activated protein kinase signal transduction pathways in the mouse brain upon infection with neurovirulent influenza A virus. J Gen Virol 2003, 84:2401-2408.

16. Takahashi M, Yamada T, Nakajima S, Nakajima K, Yamamoto T, Okada H: The substantia nigra is a major target for neurovirulent influenza A virus. J Exp Med 1995, 181:2161-2169.

17. Liu HB, Lisa G, Eugene CD, Robert MK, Ronald T, Andrew PR: The ESEV PDZ Binding-Motif of the Avian Influenza A Virus NS1 Protein Protects Infected Cells from Apoptosis through Directly Targeting Scribble. J Virol 2010, 10:1278-1288.

18. Benjamin GH, Richard ER, David J, Juan O, David J: The multifunctional NS1 protein of influenza A viruses. J Gen Virol 2008, 89:2359-2376.

19. Filip J, Arkadiusz O, Katarzyna S, Jacek O: PDZ domains-common players in the cell signaling. ABP Vol 2003, 50:985-1017.

20. Walter DV, Joost H, Ron F, Peter DH, Ben B: Differential RNA silencing suppression activity of NS1 proteins from different influenza A strains. J Gen Virol 2009, 90:1916-1922.

21. Alto NM, Shao F, Lazar CS, Brost RL, Chua G: Identification of a bacterial type III effector family with $\mathrm{G}$ protein mimicry functions. Cell 2006, 124:133-145.

22. Kochs G, Garcia-Sastre A, Martinez-Sobrido L: Multiple anti-interferon actions of the influenza A virus NS1 protein. J Virol 2007, 81:7011-7020.

23. Isamu M, Fumi G, Yoshinori I, Shinichi K: Olfactory receptor neurons prevent dissemination of neurovirulent influenza $A$ virus into the brain by undergoing virus-induced apoptosis. J Gen Virol 2002, 83:2109-2116.

24. Doorbar J: Molecular biology of human papillomavirus infection and cervical cancer. Clin Sci 2006, 110:525-541.

25. David J, Jaber MD, Danielle H: A new influenza virus virulence determinant: The NS1 protein four C-terminal residues modulate pathogenicity. PNAS 2008, 105:4381-4386.

26. Nikonenko I: Protein shown to be major component of synapse construction. J Cell Biol 2008, 10:1083-1092.

27. Dash PR, Cartwright JE, Baker PN, Johnstone AP, Whitley GS: Nitric oxide protects human extravillous trophoblast cells from apoptosis by a cyclic GMP-dependent mechanism and independently of caspase 3 nitrosylation. Exp Cell Res 2003, 287:3114-3124.

28. Gilbert AB, Dominique $G$, Marius $G$, Simon A: Nitric oxide, cell signaling and cell death. Toxicology 2005, 208:177-192.

29. Matsunaga T, Kotamraju S, Kalivendi SV, Dhanasekaran A, Joseph J, Kalyanaraman B: Ceramide-induced intracellular oxidant formation, iron signaling, and apoptosis in endothelial cells: protective role of endogenous nitric oxide. J Biol Chem 2004, 279:28614-28624.

30. Tabuchi A, Oh E, Taoka A, Sakurai H, Tsuchiya T, Tsuda M: Rapid attenuation of AP-1 transcriptional factors associated with nitric oxide (NO)-mediated neuronal cell death. J Biol Chem 1996, 271:31061-31067.

31. Fitzsimonds RM, Poo MM: Retrograde signaling in the development and modification of synapses. Physiol Rev 1998, 78:143-170.

doi:10.1186/1743-422X-8-298

Cite this article as: Zhang et al:: The distinct binding properties between avian/human influenza A virus NS1 and Postsynaptic density protein-95 (PSD-95), and inhibition of nitric oxide production. Virology Journal 2011 8:298. 